\title{
Efecto de la Liofilización de Brócoli Pre-Procesado sobre la Cinética de Secado y el Contenido de Sulforafano
}

\author{
Andrea V. Mahn ${ }^{(1) \star}$, Juan I. Román ${ }^{(2)}$ y Alejandro E. Reyes ${ }^{(1)}$ \\ (1) Departamento de Ingeniería Química, Universidad de Santiago de Chile, Avenida Libertador Bernardo \\ O’Higgins 3363, Estación Central, Santiago, Chile. (e-mail: andrea.mahn@usach.cl) \\ (2) Programa Doctorado en Ciencia y Tecnología de los Alimentos, Universidad de Santiago de Chile, \\ Obispo Manuel Umaña 050, Estación Central, Santiago, Chile.
}

${ }^{*}$ Autor a quien debe ser dirigida la correspondencia

Recibido Mar. 11, 2016; Aceptado Abr. 26, 2016; Versión final May. 16, 2016, Publicado Dic. 2016

\section{Resumen}

Se estudió la liofilización de bloques de brócoli pre-procesado mediante un diseño factorial $3^{1} \cdot 2^{1}$ para investigar el efecto del espesor de los bloques $(5 \mathrm{~mm} ; 7.5 \mathrm{~mm} ; 10 \mathrm{~mm}$ ) y la velocidad de congelación (alto: nitrógeno líquido; bajo: congelador doméstico) sobre la cinética de secado, contenido de humedad y contenido de sulforafano. El contenido de sulforafano fue máximo para bloques de $7.5 \mathrm{~mm}$ y velocidad de congelación alta, obteniéndose un contenido de sulforafano $29.75 \%$ [3.057 $\mu \mathrm{mol} / \mathrm{g}$ materia seca] mayor que la condición previa al secado, que corresponde a brócoli pre-procesado (escaldado e incubado) en condiciones óptimas [2.356 $\mu \mathrm{mol} / \mathrm{g}$ materia seca]. De acuerdo al estudio de superficie de respuesta, las condiciones óptimas para obtener brócoli liofilizado con alto contenido de sulforafano son espesor de 8.3 $\mathrm{mm}$ y congelación rápida, alcanzando un contenido de sulforafano igual a 3.02 [ $\mu \mathrm{mol} / \mathrm{g}$ materia seca]. Se completó el periodo de secado primario sólo para espesor de 5.0 y $7.5 \mathrm{~mm}$. Se ajustaron las curvas de secado al modelo de difusividad constante simplificado dando una difusividad efectiva entre $7.4 \cdot 10^{-11} \mathrm{y}$ $1.23 \cdot 10^{-10}\left[\mathrm{~m}^{2} / \mathrm{s}\right]$. Las curvas de secado fueron ajustadas a tres modelos empíricos (Page, Weibull y Logaritmico). Los mejores ajustes los entregaron el modelo de Page y el modelo Logarítmico $\left(R^{2} \geq 0.995\right)$.

\section{Effect of Freeze-Drying of Pre-Processed Broccoli on Drying Kinetics and Sulforaphane Content}

\begin{abstract}
Freezer-drying of pre-processed broccoli blocks were studied through a $3^{1} \cdot 2^{1}$ factorial design to investigate the effect of the broccoli blocks thickness $(5 \mathrm{~mm} ; 7.5 \mathrm{~mm} ; 10 \mathrm{~mm}$ ) and freezing rate (high: immersion in liquid nitrogen; low: household freezer) on drying kinetics, sulforaphane and moisture content. The maximum sulforaphane content was achieved with $7.5 \mathrm{~mm}$ thickness and high freezing rate. In this condition, sulforaphane content was increased by $29.75 \%$ [3.057 $\mu \mathrm{mol} / \mathrm{g}$ dry matter] compared to the situation before drying, corresponding to pre-processed broccoli (blanched and incubated) in optimal conditions [2.356 $\mu \mathrm{mol} / \mathrm{g}$ dry matter]. According to the response surface analysis, the optimal conditions to obtain freeze-dried broccoli are $8.3 \mathrm{~mm}$ thickness and high freezing rate, resulting in a sulforaphane content equal to $3.02[\mu \mathrm{mol} / \mathrm{g}$ dry matter]. The primary drying period was completed only for thickness of 5.0 and 7.5 $\mathrm{mm}$. Adjustment of drying curves to the simplified constant diffusivity model gave effective moisture diffusivity values between $7.4 \cdot 10^{-11}$ and $1.23 \cdot 10^{-10}\left[\mathrm{~m}^{2} / \mathrm{s}\right]$. The drying curves were adjusted with three empirical models (Page, Weibull y Logarithmic). The best fittings were obtained with the Page and the Logarithmic models $\left(R^{2} \geq 0.995\right)$.
\end{abstract}

Keywords: freeze-drying, broccoli, sulforaphane, drying kinetics, optimization 


\section{INTRODUCCIÓN}

El brócoli (Brassica oleracea var. italica) ofrece múltiples beneficios para la salud, al contener compuestos antioxidantes, vitaminas, minerales, selenio y glucosinolatos (Mahn y Reyes, 2012a; Moreno et al., 2006). Los glucosinolatos, son los precursores de los isotiocianatos, de los cuales el sulforafano es el más relevante, porque posee propiedades antimicrobianas, antioxidantes y anticarcinogénicas comprobadas in vitro e in vivo (Fimognari y Hrelia, 2007; Campas-Baypoli et al., 2009). El brócoli es consumido como un alimento procesado, lo que afecta el contenido de compuestos bioactivos. Algunos procesos, tales como el escaldado, la cocción, el congelación y la deshidratación, afectan el contenido de glucosinolatos, polifenoles y sulforafano (Bestard et al., 2001; Wang et al, 2012). Se ha reportado una pérdida de un $30 \%$ de los glucosinolatos totales al escaldar brócoli fresco, y una pérdida de $72.4 \%$ de los glucosinolatos totales al cocer brócoli fresco (Cieślik et al., 2007). Por otra parte, otros estudios señalan que un proceso adecuado de escaldado incrementa el contenido de sulforafano, con respecto al brócoli fresco (Wang et al., 2012; Pérez et al. 2014).

Además del escaldado y la cocción, el brócoli puede ser procesado para su preservación, mediante congelación, refrigeración o deshidratación. La congelación a temperaturas inferiores a $-18^{\circ} \mathrm{C}$ representa un alto costo en la industria alimenticia, debido a la necesidad de mantener la cadena de frío. En tanto la refrigeración a $4^{\circ} \mathrm{C}$, permite preservar los alimentos durante un tiempo limitado. Aires et al. (2012) determinaron el efecto de las condiciones de almacenamiento en algunas Brassicas, y señalaron que la refrigeración afecta negativamente el contenido de glucosinolatos con posterioridad a las 72 horas de almacenamiento a $4^{\circ} \mathrm{C}$. Por lo tanto, durante el almacenamiento en condiciones de refrigeración se producen pérdidas de propiedades bioactivas del brócoli.

La deshidratación es un método ampliamente usado para preservar alimentos. El efecto de las condiciones de secado sobre los compuestos bioactivos del brócoli en secado convectivo en secadores de túnel y de lecho fluidizado ha sido documentado. En experiencias de deshidratación de brócoli en un secador de túnel un aumento de la temperatura del aire y del tamaño del sustrato afectó negativamente la capacidad antioxidante del vegetal (Mahn et al., 2011). Reyes et al. (2012), estudiaron el secado de inflorescencias de brócoli en un secador de lecho fluidizado, y reportaron que el proceso no afectó la capacidad antioxidante del vegetal, pero sí disminuyó el contenido de selenio en un $35 \%$ aproximadamente. En tanto, existen pocos estudios acerca del secado de brócoli por liofilización. Mahn et al. (2012b) investigaron el proceso de liofilización de brócoli enriquecido en selenio, concluyendo que la liofilización a presión atmosférica maximiza el contenido de selenio orgánico en el producto final. Sin embargo, el contenido de polifenoles y la capacidad antioxidante decrecieron significativamente en relación al vegetal fresco.

Recientemente, se desarrolló una sopa rica en sulforafano. En el estudio se compararon dos tipos de formulaciones, sopa enriquecida con tallos de brócoli liofilizados y sopa enriquecida con inflorescencias de brócoli liofilizadas, con una sopa de brócoli comercial, usada como control. Las formulaciones se sometieron a un proceso de cocción por microondas, midiéndose el contenido de sulforafano. En la sopa enriquecida con inflorescencias se obtuvo un contenido de sulforafano de 0.2 [ $\mu \mathrm{mol} / \mathrm{g}$ sopa seca], mientras que ni en la sopa comercial ni en la sopa enriquecida con tallos de brócoli se detectó este compuesto (Alvarez-Jubete et al., 2014). Estos valores son significativamente inferiores que los reportados por Pérez et al. (2014), quienes optimizaron el proceso de escaldado de inflorescencias de brócoli logrando un contenido máximo de sulforafano igual a $4.0[\mu \mathrm{mol} / \mathrm{g}$ materia seca].

En consecuencia, en este trabajo se propone que la liofilización de brócoli previamente sometido a escaldado e incubado en condiciones óptimas permitiría obtener un producto deshidratado con alto contenido de sulforafano. Actualmente, no hay estudios que muestren el efecto de las condiciones de liofilización sobre el contenido de sulforafano en brócoli. El objetivo de este trabajo fue investigar el efecto de la liofilización de brócoli pre-procesado sobre el contenido de sulforafano y la cinética de secado, incluyendo el ajuste a modelos matemáticos.

\section{Cinética de secado}

La cinética de secado está determinada por la difusión de la humedad desde el sustrato hacia el aire de secado. Se puede describir mediante la segunda ley de Fick (Ec. 1), donde X representa el contenido de humedad y $D_{\text {eff }}$ es el coeficiente de difusividad efectiva (Crank, 1975).

$$
\frac{\partial X}{\partial t}=D_{\text {eff }} \frac{\partial^{2} X}{\partial z^{2}}
$$

La Ec. 1 se resuelve según la geometría estudiada y las condiciones de borde iniciales. En este trabajo se 
consideró que el sustrato posee una humedad inicial uniforme, sólo existe resistencia interna para la transferencia de masa, no existe encogimiento del sólido durante el proceso de secado, se desprecian los efectos internos y externos para la transferencia de calor y la difusividad efectiva permanece constante durante todo el proceso. Con estos supuestos, la solución matemática expresada para la humedad promedio en todo el sólido, está dada por la Ec. 2.

$$
\frac{X(t)-X_{e q}}{X_{0}-X_{e q}}=\sum_{n=1}^{\infty} \frac{2 v}{\lambda_{n}{ }^{2}} \exp \left[-\lambda_{n}{ }^{2} \frac{D_{e f f}}{a^{2}} \cdot t\right]
$$

Considerando que el parámetro 'a' es el semiespesor del bloque, $v$ y $\lambda_{n}$ son definidos de acuerdo a la geometría del sustrato, para una placa plana infinita y considerando sólo el primer término de la sumatoria, se obtiene el modelo de difusividad constante simplificado (MDCS) (Ec. 3).

$$
\frac{X}{X_{0}}=\exp \left(-\frac{\pi^{2} \cdot D_{e f f} \cdot t}{4 \cdot a^{2}}\right)
$$

Debido a la simplicidad del modelo, adaptable a distintas geometrías, éste resulta una herramienta útil para describir la cinética de secado. La modelación matemática del proceso de secado es necesaria tanto para el diseño de equipos y procesos, como para la optimización, control y automatización de éstos (Rayaguru, 2012). Además, el uso de modelos matemáticos empíricos, permite estimar el tiempo necesario para alcanzar el contenido de humedad deseado, y permite estudiar la eficiencia del proceso (Meisami, 2009).

En este trabajo se consideraron los modelos empíricos de Page (Ec. 4), Logarítmico (Ec. 5) y Weibull (Ec. 6), que derivan de la segunda ley de Fick y muestran una relación directa entre el contenido de humedad y el tiempo de secado (Rayaguru, 2012). El modelo de Page se muestra en la Ec. 4 (Page, 1949), donde k $\left[\mathrm{min}^{-1}\right]$ y $\mathrm{n}$ (adimensional) corresponden a constantes de ajuste.

$$
\frac{X}{X_{0}}=\exp \left(-k \cdot t^{n}\right)
$$

El modelo Logarítmico se muestra en la Ec. 5, donde b, $\mathrm{k}$ y $\mathrm{c}$ son constantes de ajuste adimensionales (Togrul 2002).

$$
\frac{X}{X_{0}}=b \cdot[\exp (-k \cdot t)]+c
$$

La Ec. 6 muestra el modelo de Weibull, donde b y c son constantes de ajuste adimensionales (Corzo, 2008).

$$
\frac{X}{X_{0}}=\exp \left(-(t / b)^{c}\right)
$$

\section{METODOLOGÍA}

Se plantea el diseño experimental, se especifica la materia vegetal usada, se explica el proceso de liofilización, se presenta la forma en que se determinó el contenido de sulforafano. Luego se presentan los modelos matemáticos usados y el análisis estadístico.

\section{Diseño experimental}

Se utilizó un diseño factorial multinivel $3^{1} \cdot 2^{1}$ en dos bloques, cuyos factores experimentales fueron: espesor de los bloques de brócoli pre-procesado $(5 \mathrm{~mm} ; 7.5 \mathrm{~mm} ; 10 \mathrm{~mm}$ ) y velocidad de congelación (alto: inmersión en nitrógeno líquido; bajo: en congelador doméstico). Las respuestas analizadas fueron el contenido de humedad y de sulforafano de las muestras después de 11 horas de liofilización. La tabla 1 muestra la matriz experimental en orden estándar. 


\section{Material vegetal}

El brócoli fue comprado a un único proveedor (Lo Valledor, Santiago, Chile) para obtener una materia prima homogénea y fresca. Se descartaron las hojas y los tallos, y las inflorescencias fueron cortadas en trozos de un largo de 5 a $7 \mathrm{~cm}$. El brócoli fresco (dos días post-cosecha) se sometió a un proceso de escaldado en condiciones optimizadas en trabajos anteriores (Pérez et al., 2014), consistentes en la inmersión en agua a $57^{\circ} \mathrm{C}$ por $13 \mathrm{~min}$.

Tabla 1: Matriz experimental en orden estándar

\begin{tabular}{|c|c|c|}
\hline Corrida & Espesor $(\mathrm{mm})$ & Velocidad de Congelación \\
\hline 1 & 5 & Lento \\
\hline 2 & 7.5 & Lento \\
\hline 3 & 10 & Lento \\
\hline 4 & 5 & Rápido \\
\hline 5 & 7.5 & Rápido \\
\hline 6 & 10 & Rápido \\
\hline
\end{tabular}

Posteriormente, el brócoli escaldado se trituró e incubó mediante inmersión en agua a $40^{\circ} \mathrm{C}$ por una hora, para lograr la máxima conversión de glucorafanina en sulforafano (Pérez, 2015). Luego, la pasta de brócoli ya escaldada, triturada e incubada, que corresponde al brócoli pre-procesado, fue moldeada como prismas rectangulares para conseguir el espesor y geometría deseados, Fig. (1). La humedad del brócoli preprocesado en forma de pasta fue de $90 \%$ en base húmeda $(X=9$ en base seca). Los bloques fueron congelados de manera lenta a $-18^{\circ} \mathrm{C}$ (congelador doméstico) durante $24 \mathrm{~h}$ o de manera rápida por inmersión en nitrógeno líquido a $-192^{\circ} \mathrm{C}$ durante $3 \mathrm{~min}$. Finalmente, los bloques de brócoli se liofilizaron en un liofilizador a vacío comercial.

(A)
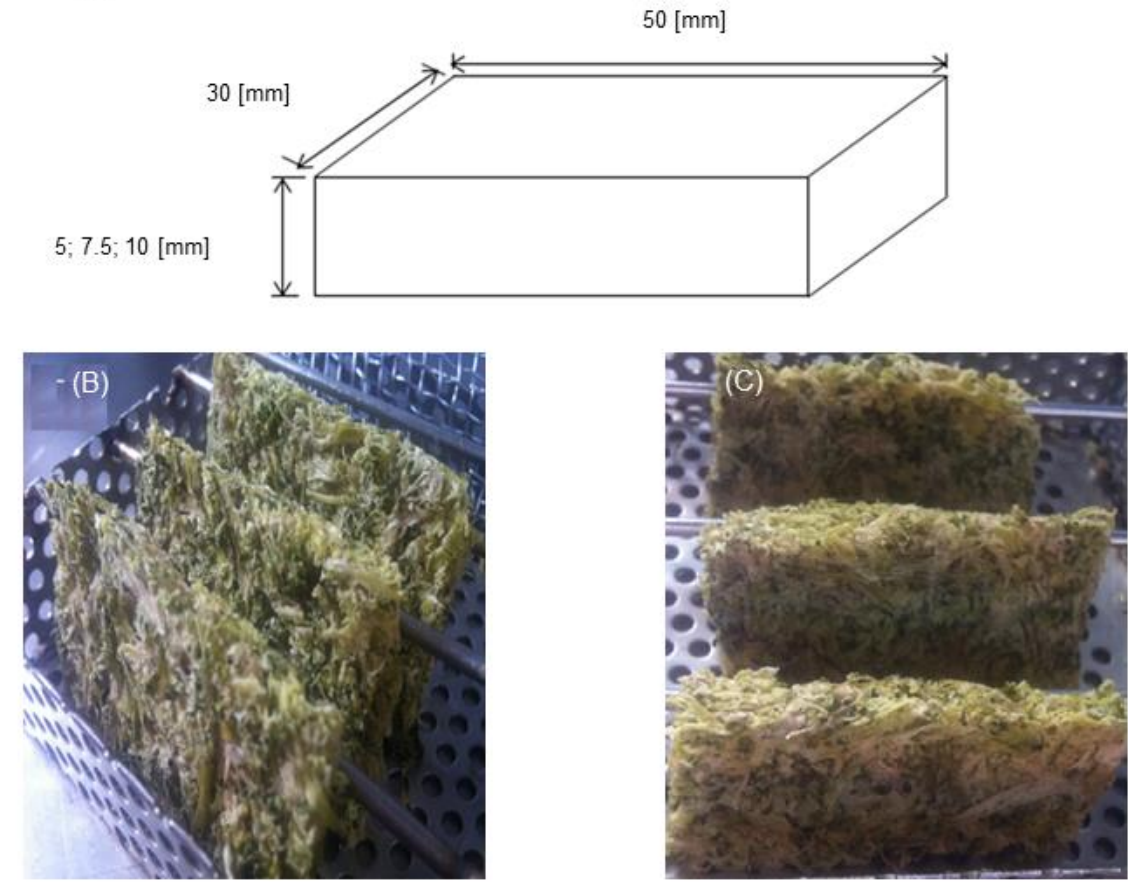

Fig. 1: (A) Esquema con las dimensiones de los bloques de brócoli. (B) y (C) Imágenes de bloques de brócoli.

\section{Liofilización}

Las corridas fueron realizadas en un liofilizador comercial marca Labconco modelo FreeZone 4.5L (Kansas, $\mathrm{MI}$, USA). La temperatura del condensador fue fijada a $-48^{\circ} \mathrm{C}$ y la presión interna de la cámara fue de $60 \mathrm{~Pa}$. Las muestras de brócoli pre-procesado fueron puestas dentro de recipientes de vidrio de $375 \mathrm{~mL}$, y la masa inicial fue medida usando una balanza analítica (Precisa Instruments, AG, Switzerland). Durante las 
primeras 4 horas las muestras se pesaron cada 30 minutos, y después se pesó cada una hora, hasta alcanzar peso constante (11 horas para la mayoría de las corridas). El contenido de humedad se determinó en una estufa a vacío (modelo G05053-12, Cole \& Palmer) según métodos estándar (AOAC, 1990), en duplicado.

\section{Contenido de Sulforafano}

El contenido de sulforafano se determinó mediante HPLC de fase reversa, según el método propuesto por Liang et al. (2006), con algunas modificaciones (Pérez et al., 2014). Se pesó 1 gramo de cada una de las muestras de brócoli, se extrajo en $10 \mathrm{~mL}$ de cloruro de metileno adicionando con $0.5 \mathrm{~g}$ de Na $\mathrm{SO}_{4}$ anhidro. La mezcla se filtró con papel filtro para separar residuos vegetales y el remanente de cloruro de metileno se secó a $30^{\circ} \mathrm{C}$ bajo vacío en un evaporador rotatorio (modelo RE300, Stuart). Luego el residuo se disolvió en $1 \mathrm{~mL}$ de acetonitrilo y se filtró con un filtro de jeringa de 0.22 micras, para finalmente inyectarlo en el HPLC.

\section{Modelos matemáticos}

Para ajustar los datos experimentales de secado a los modelos empíricos (modelo Page, Logarítmico y Weibull), y al modelo fenomenológico (modelo de difusividad constante simplificada), se utilizó el método de Newton, usando derivadas progresivas y estimación lineal, en el programa Microsoft Excel®, en donde se minimizó la sumatoria de los residuos cuadráticos (RSS), y la calidad del ajuste se determinó utilizando el error cuadrático medio (RMSE) y el coeficiente de determinación $\left(R^{2}\right)$. Para ello se utilizaron las Ecs. 7 y 8 : La bondad del ajuste se evaluó considerando el $R^{2}$ más cercano a uno, y valores de RMSE más bajos (Trogrul, 2002; Meisami, 2009).

$R S S=\sum_{i=1}^{n}\left(X_{\exp i}-X_{\bmod i}\right)^{2}$

$R M S E=\sqrt{\frac{\sum_{i=1}^{n}\left(X_{\exp \dot{ }}-X_{\bmod j}\right)^{2}}{N}}$

\section{Análisis estadístico}

Los efectos estandarizados de los factores experimentales y su significancia (95\% de confianza) fueron calculados de acuerdo a procedimientos estándar (Box, Hunter, \& Hunter, 1989), usando el programa Statgraphics $^{\text {TM }}$ Centurion XVII (Statistical Graphics Corp., USA, 2013). Las diferencias estadísticamente significativas entre el brócoli antes y después de liofilizado se determinaron mediante la prueba t de Student con un $95 \%$ de confianza. Se aplicó la prueba de Tukey para identificar las muestras que diferían significativamente entre sí.

\section{RESULTADOS Y DISCUSION}

Se presentan los resultados de la cinética de secado, se discuten el modelado matemático de las curvas de secado, se analizan los resultados sobre contenido de sulforafano y finalmente se discute la optimización del proceso de liofilización

\section{Cinética de Secado}

En liofilización, se acepta que el secado primario de alimentos concluye cuando el sustrato alcanza entre 5 y $20 \%$ de humedad en base húmeda ( $\mathrm{X} / \mathrm{X}_{0}$ entre 0.06 y 0.22 en base seca), dependiendo de las características del alimento. En el caso del brócoli, debido a su estructura altamente fibrosa, se puede considerar que el secado primario concluye cuando $X / X_{0}$ es igual a 0.22 (base seca). En la Fig. (2) se muestra la cinética de secado de las seis corridas experimentales. Se graficó el contenido de humedad adimensional $\left(X / X_{0}\right)$ (en base seca) de los bloques de brócoli, en función del tiempo. En las curvas se observó un periodo de ajuste hasta los 60 minutos, en donde el sustrato a secar se adaptó a las condiciones presentes dentro del sistema. A continuación, en las corridas 1, 2, 4 y 5 , fue posible observar el periodo de secado primario, que finalizó después de los $480 \mathrm{~min}$ para un espesor de $5 \mathrm{~mm}$ y después de $540 \mathrm{~min}$ para un espesor de $7.5 \mathrm{~mm}$. En las corridas 3 y 6 no se completó el secado primario, ya que la humedad adimensional final fue mayor que 0.22 . Esto se debe probablemente a que el camino que debe recorrer la humedad para salir del sustrato es más largo, y por lo tanto se requieren tiempos de secado mayores. En el periodo de secado primario se removió gran parte de la humedad presente en el vegetal. En el tramo de 
secado secundario, donde la velocidad de secado se reduce, se removió agua desde el centro del bloque de brócoli, la cual debió atravesar la capa de sólido seco, lo que dificultó la transferencia de masa de agua. El contenido de humedad final que se alcanzó en las distintas corridas fue relativamente elevado $\left(X / X_{0}\right.$ final fluctuó entre 0.11 y 0.28 , en base seca), debido al largo periodo de tiempo necesario para completar el secado primario requerido en liofilización de productos fibrosos. Nuestros resultados coinciden con Yang et al. (2013) para liofilización de brócoli.

Se ha reportado que los periodos de tiempo necesarios en liofilización para alcanzar contenidos de humedad que aseguren la estabilidad del producto son prolongados. Reyes et al. (2011) reportó que para la liofilización a vacío de Concholepas concholepas, se obtuvo una fase de secado primario de 4 horas y un secado secundario hasta las 12 horas aproximadamente. Un estudio realizado por Vázquez-Chávez et al. (2008) sobre secado de trigo, señaló que existe una capa superficial en el endospermo del trigo, que controla la velocidad de eliminación de agua, haciendo una deshidratación más lenta en el tramo de secado secundario. Del mismo modo, un estudio hecho por Hernández-Díaz et al. (2013), sobre el secado de granos de café, mostró que el periodo de secado secundario, después del contenido de humedad critica (0.17 kg de agua $/ \mathrm{kg}$ materia seca), necesita más energía para remover el agua ligada, además se forma una barrera a la transferencia de calor y materia, debida a la creciente capa de sólido seco. Los autores señalaron que esto influye en la eficiencia térmica y energética del secado.
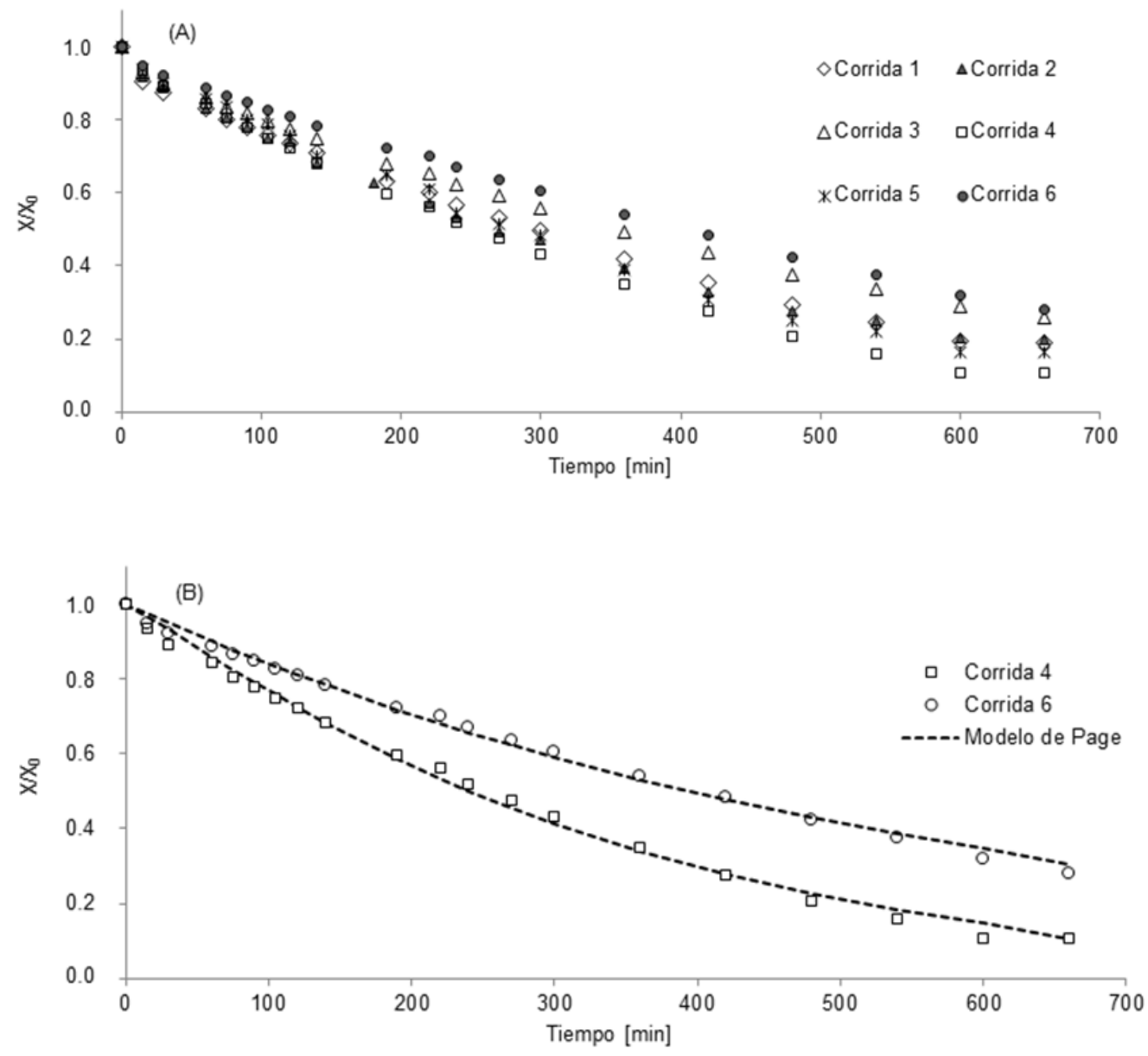

Fig. 2: Cinética de secado del proceso de liofilización, de las corridas experimentales descritas en la tabla 1. (A): datos experimentales, (B): ajuste de las corridas 4 y 6 al el modelo de Page.

En este trabajo se estudiaron los efectos del espesor y la velocidad de congelación y su significancia estadística, sobre el contenido de humedad, para 660 minutos de secado en todas las corridas experimentales. En el diagrama de Pareto mostrado en la Fig. (3), se observan los efectos significativos de los factores estudiados. En la figura 3, Barras rellenas indican un efecto positivo, barras vacías indican un efecto negativo, sobre el contenido de humedad. (B) Gráfico de superficie de respuesta para el contenido de humedad final. El efecto del espesor $(p=0.0000)$, resultó estadísticamente significativo, al igual que la velocidad de congelación ( $p=0.0022$ ). Un aumento en el espesor, de $5 \mathrm{~mm}$ a $10 \mathrm{~mm}$, generó un aumento significativo en el contenido de humedad de 0.15 a 0.30 [ $\mathrm{kgH}_{2} \mathrm{O} / \mathrm{kgb}$.s.], mientras que un aumento en la velocidad de congelación, redujo significativamente el contenido de humedad de 0.20 a $0.16\left[\mathrm{kgH}_{2} \mathrm{O} / \mathrm{kgb}\right.$.s.]. 
De acuerdo a los resultados obtenidos, las condiciones que entregan un menor contenido de humedad al cabo de 11 horas de secado corresponden a espesor $5 \mathrm{~mm}$ y velocidad de congelación rápida (con nitrógeno líquido) (corrida 4). Este resultado concuerda con lo esperado, ya que un menor espesor implica una ruta de salida de humedad más corta, y por lo tanto menor tiempo de secado.

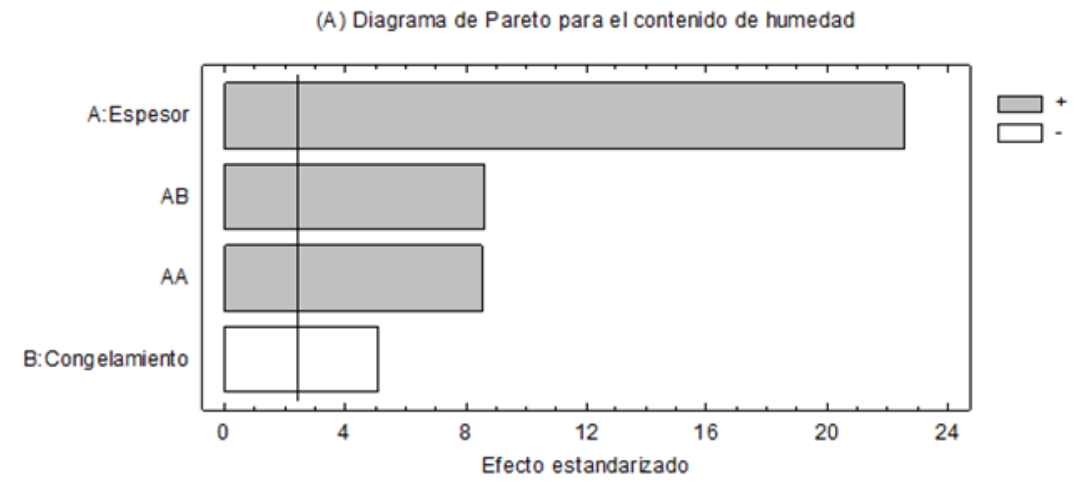

(B) Superficie de respuesta estimada

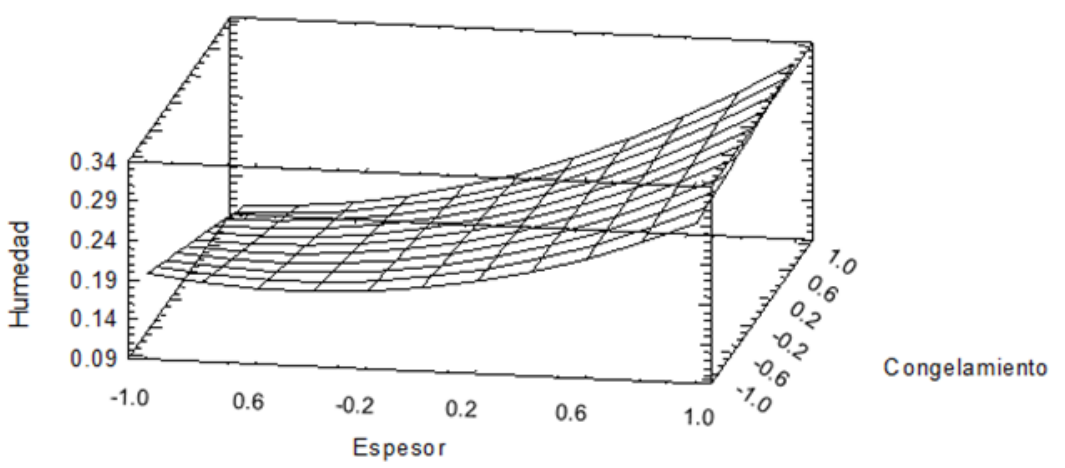

Fig. 3: Diagrama de Pareto que muestra la significancia de los efectos de los factores experimentales.

\section{Modelado matemático de las curvas de secado}

Las curvas de secado se ajustaron a un modelo fenomenológico (MDCS) basado en la segunda ley de Fick, y a tres modelos empíricos (modelo de Page, modelo Logarítmico y modelo de Weibull) para las diferentes condiciones estudiadas. En la tabla 2 se muestran las constantes de ajuste de cada modelo y los parámetros estadísticos, para las 6 corridas experimentales.

Se puede observar que la difusividad efectiva $\left(D_{\text {eff }}\right)$ varió en el rango de $7.4 .10^{-11}$ a $1.23 .10^{-10}\left[\mathrm{~m}^{2} / \mathrm{s}\right]$. Estos valores son dos órdenes de magnitud inferiores a los reportados por Doymaz y Sahin (2016) para secado de láminas de brócoli con aire caliente $\left(3.27 .10^{-9}-1.23 \cdot 10^{-8}\left[\mathrm{~m}^{2} / \mathrm{s}\right]\right)$, y un orden de magnitud inferiores que los reportados por Mahn et al. (2011) para secado de inflorescencias de brócoli en un secador de bandejas. Doymaz (2014) estudió el secado convectivo de brócoli, obteniendo una $D_{\text {eff }}$ que fluctuó entre $1.987 .10^{-8}$ y $3.577 .10^{-8}\left[\mathrm{~m}^{2} / \mathrm{s}\right]$. Estas diferencias en la difusividad efectiva pueden atribuirse a los diferentes métodos de secado usados por los distintos autores y a la dependencia que existe entre la difusividad efectiva y temperatura de secado. En un estudio realizado en el secado convectivo del alga gracilaria chilensis, se demostró que al aumentar la temperatura de secado, la difusividad efectiva aumenta proporcionalmente (Vega et al., 2007). Los resultados obtenidos en el presente trabajo coinciden en orden de magnitud con los reportados por Reyes et al. (2011), para la liofilización de rebanadas de manzana, en donde la difusividad efectiva fluctuó entre $7.12 .10^{-11}$ a $6.76 \cdot 10^{-10}\left[\mathrm{~m}^{2} / \mathrm{s}\right]$.

En cuanto a los modelos empíricos, se observó que tanto el modelo de Page como el modelo Logarítmico ajustaron satisfactoriamente a las curvas de secado experimentales, dando $R^{2}$ mayores o iguales a $99.5 \%$ y RMSE en promedio igual a 0.03. De los cuatro modelos estudiados, el modelo de Page es el más utilizado en la industria alimentaria, debido a su simplicidad y a la buena calidad de ajuste que entrega, como queda demostrado en los trabajos de Michalewicz et al. (2011) para el ajuste de curvas de secado de cajuil en un secador de bandejas, y de Vega y Lemus (2006) para secado de papaya con aire caliente. En la Fig. (2B), se muestra el ajuste dado por el modelo de Page, para las corridas 4 y 6 . Se evidencia que para ambas corridas se obtiene un muy buen ajuste $\left(R^{2}>99.7 \%\right)$. Estos resultados concuerdan con Reyes et al. (2011), 
quienes reportaron que el modelo empírico de Page se ajustó a las curvas de secado con un $\mathrm{R}^{2}$ superior a 90\%. Doymaz (2014) investigó el ajuste de 12 modelos empíricos, entre los cuales el modelo que mejor se ajustó a los datos fue el modelo de Midilli, obtenido a partir de la segunda ley de Fick, con cuatro constantes de ajuste (Midilli, 2002).

Tabla 2: Constantes de ajuste y parámetros estadísticos, de los modelos MDCS, Page, Logarítmico y Weibull, para las 6 corridas experimentales.

\begin{tabular}{|c|c|c|c|c|c|c|c|c|c|}
\hline Corrida & Modelo & $D_{\text {eff }}\left[\mathrm{m}^{2} / \mathrm{s}\right]$ & $\mathrm{k}\left[\mathrm{s}^{-1}\right]$ & $n$ & $\mathrm{~b}$ & c & RSS & RMSE & $\mathrm{R}^{2}$ \\
\hline \multirow[t]{4}{*}{1} & MDCS & $1.05 \mathrm{E}-10$ & - & - & - & - & 0.012 & 0.025 & 0.996 \\
\hline & Page & - & 0.159 & 0.961 & - & - & 0.011 & 0.024 & 0.995 \\
\hline & Logarítmico & - & 0.142 & - & 0.970 & $3.1 \mathrm{E}-05$ & 0.008 & 0.020 & 0.996 \\
\hline & Weibull & - & - & - & 0.438 & 0.078 & 0.022 & 0.032 & 0.995 \\
\hline \multirow[t]{4}{*}{2} & MDCS & $1.11 \mathrm{E}-10$ & - & - & - & - & 0.006 & 0.017 & 0.998 \\
\hline & Page & - & 0.166 & 0.966 & - & - & 0.005 & 0.016 & 0.998 \\
\hline & Logarítmico & - & 0.152 & - & 0.978 & 3.0E-05 & 0.004 & 0.014 & 0.998 \\
\hline & Weibull & & & & 0.475 & 0.072 & 0.014 & 0.026 & 0.996 \\
\hline \multirow[t]{4}{*}{3} & MDCS & $8.54 \mathrm{E}-11$ & - & - & - & - & 0.006 & 0.017 & 0.998 \\
\hline & Page & - & 0.134 & 0.941 & - & - & 0.004 & 0.015 & 0.998 \\
\hline & Logarítmico & - & 0.115 & - & 0.975 & 3.0E-05 & 0.003 & 0.011 & 0.998 \\
\hline & Weibull & - & - & - & 0.548 & 0.058 & 0.008 & 0.019 & 0.996 \\
\hline \multirow[t]{4}{*}{4} & MDCS & $1.23 \mathrm{E}-10$ & - & - & - & - & 0.014 & 0.027 & 0.996 \\
\hline & Page & - & 0.149 & 1.105 & - & - & 0.009 & 0.021 & 0.997 \\
\hline & Logarítmico & - & 0.177 & - & 1.008 & 3.0E-05 & 0.013 & 0.026 & 0.995 \\
\hline & Weibull & - & - & - & 0.513 & 0.035 & 0.012 & 0.029 & 0.996 \\
\hline \multirow[t]{4}{*}{5} & MDCS & $1.09 \mathrm{E}-10$ & - & - & - & - & 0.013 & 0.026 & 0.995 \\
\hline & Page & - & 0.130 & 1.110 & - & - & 0.007 & 0.020 & 0.997 \\
\hline & Logarítmico & - & 0.157 & - & 1.009 & 0.000 & 0.012 & 0.025 & 0.995 \\
\hline & Weibull & - & - & - & 0.479 & 0.061 & 0.032 & 0.072 & 0.996 \\
\hline \multirow[t]{4}{*}{6} & MDCS & $7.40 \mathrm{E}-11$ & - & - & - & - & 0.004 & 0.015 & 0.997 \\
\hline & Page & - & 0.103 & 1.015 & - & - & 0.004 & 0.014 & 0.998 \\
\hline & Logarítmico & - & 0.103 & - & 0.991 & 0.000 & 0.004 & 0.014 & 0.998 \\
\hline & Weibull & - & - & - & 0.498 & 0.034 & 0.063 & 0.041 & 0.995 \\
\hline
\end{tabular}

\section{Contenido de sulforafano}

En este estudio se usó brócoli fresco con un contenido de sulforafano de 0.295 [ $\mu \mathrm{mol} / \mathrm{g}$ materia seca]. El brócoli se escaldó por 13 minutos a $57^{\circ} \mathrm{C}$, obteniéndose $1.168[\mu \mathrm{mol} / \mathrm{g}$ materia seca] de sulforafano. A continuación el brócoli escaldado se incubó en un baño de agua a $40^{\circ} \mathrm{C}$ por una hora, obteniéndose un contenido medio de sulforafano igual a $2.356 \mu \mathrm{mol} / \mathrm{g}$ materia seca. El brócoli escaldado e incubado corresponde al brócoli pre-procesado, punto inicial previo al secado por liofilización, en donde se obtuvo el máximo contenido de sulforafano. Para investigar el efecto de la liofilización sobre el contenido de sulforafano, se comparó el contenido de sulforafano en el brócoli liofilizado con el contenido de sulforafano en el brócoli pre-procesado antes del secado. Para ello, el contenido final de sulforafano en el brócoli liofilizado se expresó como porcentaje del contenido inicial (antes de liofilizar).

En la Fig. (4) se muestra el porcentaje de sulforafano remanente en el brócoli liofilizado con respecto al contenido antes de liofilizar (brócoli pre-procesado). Las barras indican el promedio \pm la desviación estándar. Las letras distintas sobre las barras indican que los valores de las medias fueron estadísticamente diferentes entre sí $(p<0.05)$. En las corridas 2 y 6 se mantuvo el contenido de sulforafano en torno al $100 \%$ (mismo nivel antes de liofilizar), por lo tanto en estas corridas las pérdidas fueron despreciables. En la corrida 5 se obtuvo un contenido final de sulforafano $29 \%$ mayor que el control (equivalente a $3.04[\mu \mathrm{mol} / \mathrm{g}$ materia seca]). Debido a que la desviación estándar para este dato fue elevada (cercana al $30 \%$ ), no se puede afirmar con certeza si este incremento en sulforafano sería reproducible ni atribuirlo al efecto de 
condiciones de operación. En las corridas 1, 3 y 4 el contenido de sulforafano disminuyó significativamente hasta alrededor del $50 \%$ con respecto al contenido de sulforafano inicial en el brócoli incubado $(p<0.05)$. En las corridas 2 y 5 se usó el mismo espesor de los bloques de brócoli $(7.5 \mathrm{~mm})$, que es mayor al espesor de las corridas 1 y $4(5 \mathrm{~mm})$, en tanto la corrida 3 y 6 tienen igual espesor $(10 \mathrm{~mm})$, pero distinta velocidad de congelación (lenta y rápida, respectivamente).

Este comportamiento se puede explicar por el efecto combinado del espesor y la velocidad de congelación sobre el contenido de sulforafano. Existe una mayor dificultad del sulforafano para difundir dentro del sustrato cuando su estructura es menos porosa, que sería el caso del congelamiento rápido, el cual produce menos daño en las estructuras celulares y por lo tanto mínimo aumento la porosidad. Dado que en la corrida 4, que presentó una gran pérdida de sulforafano, se usó congelamiento rápido, la velocidad de congelación no es el único factor que afecta el contenido de sulforafano. Esto es avalado por el análisis estadístico, según el cual la interacción entre espesor y velocidad de congelación tienen un efecto significativo sobre el contenido de sulforafano. En el caso de las corridas 1 y 4, los bloques tenían un menor espesor, y por lo tanto el sulforafano debía recorrer un camino más corto para abandonar el sustrato. En la corrida 3, si bien los bloques tenían un espesor mayor $(10 \mathrm{~mm})$, probablemente el daño estructural producido por la congelación lenta prevaleció sobre el efecto del espesor. En las corridas 5 y 6 , donde las pérdidas de sulforafano fueron mínimas, el espesor fue mayor $(7.5 \mathrm{~mm})$ y se usó velocidad de congelación rápida, por lo tanto el efecto de ambos factores impidió la pérdida del compuesto.

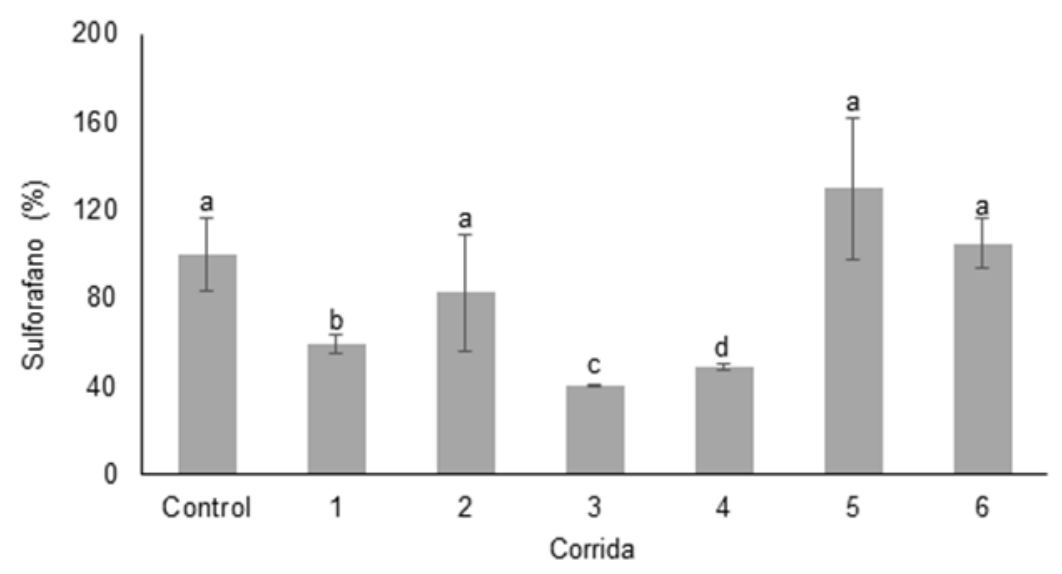

Fig. 4: Porcentaje de sulforafano en el brócoli liofilizado con respecto al contenido antes de liofilizar (brócoli escaldado e incubado).

\section{Optimización del proceso de liofilización}

El diseño experimental se analizó para calcular los efectos estadísticos de los factores experimentales y para identificar las condiciones de espesor y velocidad de congelación que maximizan contenido de sulforafano en brócoli liofilizado. En la Fig. (5A), se muestra el diagrama de Pareto para los efectos estandarizados de velocidad de congelación, espesor y las interacciones espesor - espesor y espesor -velocidad de congelación. Se observó que la velocidad congelación y la interacción entre el espesor y velocidad de congelación ejercen un efecto positivo estadísticamente significativo sobre el contenido de sulforafano. Por su parte, la interacción espesor - espesor mostró un efecto negativo significativo sobre el contenido de sulforafano. En tanto, el espesor por sí solo no mostró un efecto significativo. Mahn et al., (2012b) estudiaron el efecto de las condiciones de liofilización de brócoli sobre el contenido de polifenoles y poder antiradical (ARP), concluyendo que la velocidad de congelación lenta mantenía estas propiedades en niveles cercanos e incluso superiores a las del alimento fresco. La velocidad de congelación lenta produce mayor daño estructural, al formarse cristales amorfos de agua congelada que se traducen en una mayor porosidad del alimento liofilizado. La mayor porosidad obtenida en estas condiciones mejora la extractabilidad de los compuestos antioxidantes, y por lo tanto, es posible detectar analíticamente una mayor concentración de estos compuestos. Según los autores, esto explicaría el hecho que la concentración de antioxidantes en el producto liofilizado fuera mayor que en el vegetal fresco en algunas condiciones de liofilización.

En la Fig. (5B) se muestra el gráfico de superficie de respuesta para el contenido de sulforafano, expresado como porcentaje con respecto al contenido de sulforafano antes de liofilizar. El contenido de sulforafano alcanzó un valor máximo dentro la región experimental, identificándose un valor de espesor óptimo (8.3 $\mathrm{mm}$ ). El modelo de regresión que describe el contenido de sulforafano en función de los factores estadísticamente significativos en niveles codificados se muestra en la Ec. 9, en donde [S] es el contenido de sulforafano, E es el espesor y $\mathrm{C}$ es la velocidad de congelación. 


$$
[S]=106.235+16.893 \cdot C-42.646 \cdot E^{2}+18.681 \cdot E \cdot C
$$

El modelo experimental fue capaz de explicar el $84.6 \%$ de la variabilidad de los datos, con un coeficiente de determinación ajustado por los grados de libertad ( $\mathrm{R}^{2}$ ajustado) igual a $75.8 \%$, indicando un buen ajuste entre los valores experimentales y predichos. El modelo predice que para un espesor de $8.3 \mathrm{~mm}$ y velocidad de congelación rápida, se obtiene un contenido de sulforafano igual a 3.02 [ $\mu \mathrm{mol} / \mathrm{g}$ materia seca], valor que coincide con el máximo obtenido en este trabajo.

(A) Diagrama de Pareto para el contenido de sulforafano

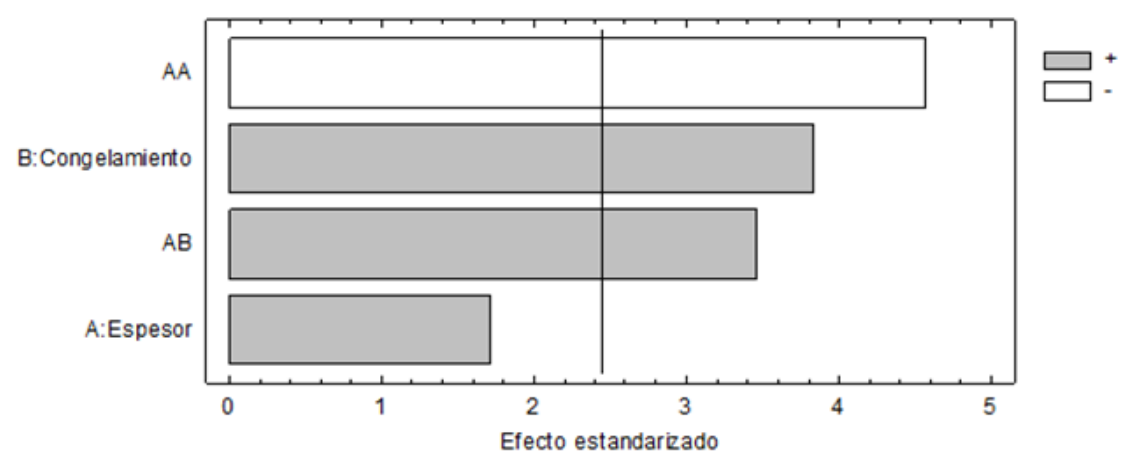

(B) Superficie de re spue sta estimada

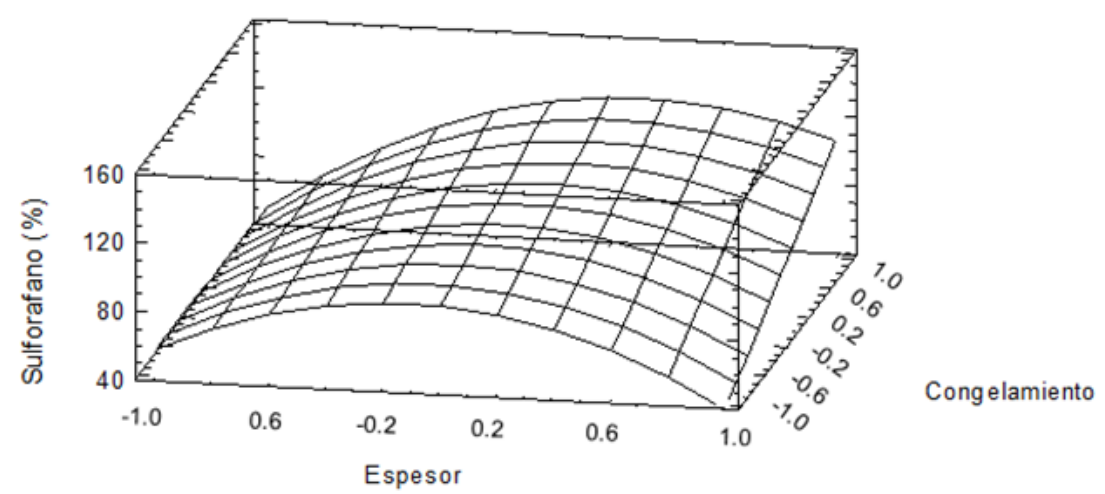

Fig. 5: (A) Diagrama de Pareto que muestra la significancia de los efectos de los factores experimentales, en el proceso de liofilización. (B) Gráfico de superficie de respuesta para el contenido de sulforafano en el brócoli liofilizado.

\section{CONCLUSIONES}

El contenido de humedad final, a los 660 minutos, fluctuó entre 0.108 y 0.282 [kg agua $/ \mathrm{kg}$ materia seco], para las diferentes corridas. El periodo de secado primario se completó sólo para espesor de 5.0 y $7.5 \mathrm{~mm}$. La difusividad efectiva $\left(D_{\text {eff }}\right)$ obtenida del ajuste del MDCS a los datos experimentales de secado se encontró entre $7.4 \cdot 10^{-11} \mathrm{y} 1.23 \cdot 10^{-10}\left[\mathrm{~m}^{2} / \mathrm{s}\right]$, lo que concuerda con lo reportado en la literatura para vegetales liofilizados. Los modelos empíricos que mejor ajustaron los datos experimentales fueron el modelo de Page y el logarítmico, con valores de $\mathrm{R}^{2}$ iguales o mayores que $99.5 \%$.

Los bloques de brócoli de espesor $7.5 \mathrm{~mm}$ y congelación rápida, correspondiente a la corrida 5 , mostraron el mayor contenido de sulforafano. En estas condiciones el contenido de sulforafano aumentó un $29.75 \%$ [3.057 $\mu \mathrm{mol} / \mathrm{g}$ materia seca] con respecto al brócoli pre-procesado, que corresponde al punto inicial, previo al secado [2.356 $\mu \mathrm{mol} / \mathrm{g}$ materia seca]. Según el estudio de superficie de respuesta, las condiciones que maximizaron el contenido de sulforafano corresponden a bloques de brócoli de $8.3 \mathrm{~mm}$ de espesor congelados por inmersión en nitrógeno líquido, alcanzando un contenido de sulforafano igual a $3.02[\mu \mathrm{mol} / \mathrm{g}$ materia seca].

\section{AGRADECIMIENTOS}

Los autores agradecen el apoyo financiero del Programa Fondecyt a través del proyecto regular $\mathrm{N}^{\circ} 1130384$ y Román J. agradece a Conicyt (Beca de estudios de doctorado, Chile). 


\section{NOTACIÓN}

\section{Símbolos}

$\begin{array}{ll}\text { a } & \text { Semiespesor de la lámina de brócoli, } \mathrm{mm} \\ \mathrm{b} & \text { Parámetro de ajuste adimensional de modelos Logarítmico y Weibull } \\ \mathrm{C} & \text { Parámetro de ajuste adimensional de modelos Logarítmico y Weibull } \\ \mathrm{D}_{\text {eff }} & \text { Difusividad efectiva, } \mathrm{m}^{2} \mathrm{~s}^{-1} \\ \mathrm{n} & \text { Parámetro de ajuste adimensional, modelo de Page } \\ \mathrm{N} & \text { Número de datos experimentales } \\ \mathrm{k} & \text { Parámetro de ajuste, modelo de Page, } \mathrm{min}^{-1} \\ \mathrm{t} & \text { tiempo, min } \\ \mathrm{X} & \text { Contenido de humedad, } \mathrm{kg} \mathrm{kg}^{-1} \\ \mathrm{X}_{0} & \text { Contenido de humedad inicial, } \mathrm{kg} \mathrm{kg}^{-1} \\ \mathrm{X}_{\text {exp,i }} & \text { Contenido de humedad, experimental } \\ \mathrm{X}_{\mathrm{mod}, \mathrm{i}} & \text { Contenido de humedad dada por el modelo }\end{array}$

\section{Abreviaturas}

$\begin{array}{ll}\text { b.s. } & \text { Base seca } \\ \text { MDCS } & \text { Modelo de difusividad constante simplificado } \\ \text { RMSE } & \text { Error cuadrático medio } \\ \text { RSS } & \text { Sumatoria de los errores al cuadrado }\end{array}$

Letras griegas

$\lambda_{\mathrm{n}} \quad$ Parámetro adimensional que para una placa infinita toma el valor de uno.

v, $\quad$ Parámetro adimensional de la segunda ley de Fick para una placa infinita.

\section{REFERENCIAS}

AOAC. Official Methods of Analysis. Association of Official Analytical Chemists, Washington, DC, USA. 15th ed.: 1298 (1990)

Aires, A., Carvalho, R. y Rosa, E. Glucosinolate Composition of Brassica is Affected by Postharvest, Food Processing and Myrosinase Activity. Journal of Food Processing and Preservation 36 (3), 214-224 (2012)

Alvarez-Jubete, L., Valverde, J. y otros cuatro autores, Development of a Novel Functional Soup Rich in Bioactive Sulforaphane Using Broccoli (Brassica oleracea L. ssp. italica) Florets and Byproducts. Food and Bioprocess Technology 7 (5), 1310-1321 (2014)

Bestard, M. J., Sanjuan, N., Rossell, C., Mulet, A., Femenia, A. Effect of storage temperature on the cell wall components of broccoli (Brassica oleracea L. Var. Italica) plant tissues during rehydration. Journal of Food Engineering 48 (4), 317-323 (2001)

Box, G. E. P., Hunter, W. G. y Hunter, J. S. Statistics for experimenters. An introduction to design, data analysis, and model building. Spain: Reverté (1989)

Campas, O.N., Bueno, C.y otros seis autores, Sulforaphane (1-isothiocyanato-4-(methylsulfinyl)-butane) content in cruciferous vegetables. Archivos Latinoamericanos De Nutricion 59 (1), 95-100 (2009)

Cieślik, E., Leszczyńska, T. Y OTROS TRES AUTORES, Effects of some technological processes on glucosinolate contents in cruciferous vegetables. Food Chemistry, 105 (3), 976-981 (2007)

Corzo, O. B., N.; Pereira, A.; Vásquez, A. Weibull distribution for modeling air drying of coroba slices. Journal of Food Science and Technology 41: 2023-2028 (2008)

Crank, J. The Mathematics of Diffusion. 2nd Edn Oxford University Press, London. (1975)

Doymaz, I. Effect of blanching temperature and dipping time on drying time of broccoli. Food Science and Technology International 20 (2), 149-157 (2014)

Doymaz, I. y Sahin, M. Effect of temperature and pre-treatment on drying and rehydration of broccoli slices. Journal of Food Measurement and Characterization (2016) in press. 
Fimognari, C. y P. Hrelia. Sulforaphane as a promising molecule for fighting cancer. Mutation Research/Reviews in Mutation Research 635 (2-3), 90-104 (2007)

Hernández-Díaz, W.N., Hernández-Campos, F.J. y otros tres autores, Optimización del secado de granos de café en un secador rotatorio. Revista Mexicana de Ingeniería Química 12, 315-325 (2013)

Liang, H., Yuan, Q. P., Dong, H.R. y Liu, Y.M. Determination of sulforaphane in broccoli and cabbage by high-performance liquid chromatography. Journal of Food Composition and Analysis 19 (5), 473-476 (2006)

Mahn, A., Antoine, P., y Reyes, A. Optimization of Drying Kinetics and Quality Parameters of Broccoli Florets. International Journal of Food Engineering 7 (2), (2011)

Mahn, A. y Reyes A. An overview of health-promoting compounds of broccoli (Brassica oleracea var. italica) and the effect of processing. Food Science and Technology International 18 (6), 503-514 (2012a).

Mahn, A., Zamorano, M. Barrientos, H. y Reyes A. Optimization of a process to obtain selenium-enriched freeze-dried broccoli with high antioxidant properties. LWT - Food Science and Technology 47 (2), 267-273 (2012b).

Meisami, E. S. Mathematical modeling of moisture content of apple slices (Var. Golab) during drying. Pakistan Journal of Nutrition 8, 804-809 (2009)

Michalewicz, J., Henriquez, J. y Charamba, J. Drying of cashew (anacardium occidentale I.): Experimental study and drying kinetics modeling. Información Tecnológica 22 (6), 63-74 (2011)

Midilli, A., Kucuk, H. y Yapar, Z. A new model for single layer drying of some vegetables. Drying Technology, 20, 1503-1513 (2002)

Moreno, D. A., Carvajal, M. López, C. y García, C. Chemical and biological characterization of nutraceutical compounds of broccoli. Journal of Pharmaceutical and Biomedical Analysis 41 (5), 1508-1522 (2006)

Page, G. E. Factors influencing the maximum rates or air drying shelled corn in thin layers. M.S. Thesis, Department of Mechanical Engineering, Purdue University, Purdue, USA (1949)

Pérez, C. Optimización del procesamiento de brócoli para maximizar el contenido de sulforafano. Tesis para optar al grado de Doctor en Ciencia y Tecnología de los Alimentos, Universidad de Santiago de Chile (2015)

Pérez, C., Barrientos, H., Román, J. y Mahn, A. Optimization of a blanching step to maximize sulforaphane synthesis in broccoli florets. Food Chemistry 145, 264-271 (2014)

Rayaguru, K. R., W. Mathematical modeling of thin layer drying kinetics of stone apple slices. International Food Research Journal 19 (4), 1503-1510 (2012)

Reyes, A., Pérez, N. y Mahn, A. Theoretical and experimental study of Freeze-drying of "loco" (Concholepas concholepas). Drying Technology 29 (12), 1386-1395 (2011)

Reyes, A., Mahn, A. Guzmán, C. y Antoniz, D. Analysis of the Drying of Broccoli Florets in a Fluidized Pulsed Bed. Drying Technology 30 (11-12), 1368-1376 (2012)

Togrul, I. T. y Pehlivan, D. Mathematical modeling of solar drying of apricots in thin layers. Food Engineering $55,209-216(2002)$

Vázquez-Chávez, L. y Vizcarra-Mendoza, M. Secado por lecho fluidizado del trigo y su calidad. Revista Mexicana de Ingeniería Química 7, 131-137 (2008)

Vega, A. y Lemus, R. Modeling of drying kinetics of chilean papaya (vasconcella pubescens). Información Tecnológica 17 (2), 47-66 (2006)

Vega, A., Tello, C., Lemus, R. Simulación matemática del proceso de secado de la gracilaria chilena (gracilaria chilensis). Revista Chilena de Ingeniería, 1 (15), 55-64 (2007)

Wang, G. C., Farnham, M. y Jeffery, E.H. Impact of Thermal Processing on Sulforaphane Yield from Broccoli (Brassica oleracea L. ssp. italica). Journal of Agricultural and Food Chemistry 60 (27), 6743-6748 (2012)

Yang, H., Yang, X. y Sun, J. Effect of different drying methods on vegetable powder quality of the broccoli (Brassica oleracea). Journal of Chinese Institute of Food Science and Technology 13 (17), 152-158 (2013) 